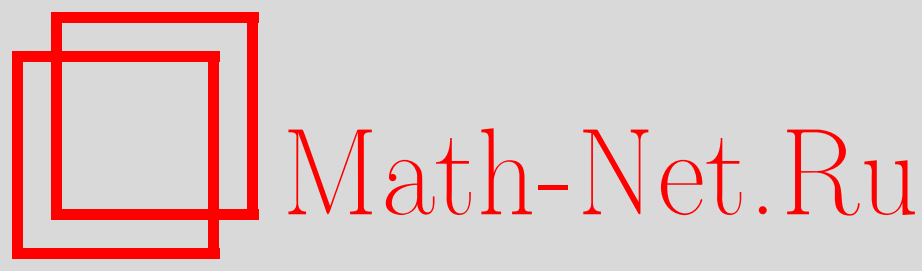

Ю. Л. Павлов, Е. В. Хворостянская, О максимальном объеме дерева в лесе Гальтона-Ватсона с ограниченным числом вершин, Дискрет. матем., 2014, том 26, выпуск $3,90-100$

DOI: https://doi.org/10.4213/dm1293

Использование Общероссийского математического портала Math-Net.Ru подразумевает, что вы прочитали и согласны с пользовательским соглашением http: //www.mathnet.ru/rus/agreement

Параметры загрузки:

IP : 34.229 .108 .108

26 апреля 2023 г., 11:28:56

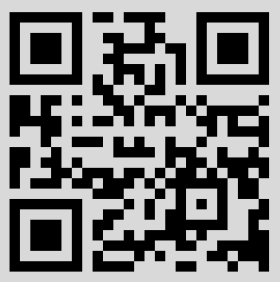




\title{
О максимальном объеме дерева в лесе Гальтона-Ватсона с ограниченным числом вершин
}

\author{
() 2014 г. Ю. Л. Павлов*, Е. В. Хворостянская**
}

Рассматривается случайный лес, образованный траекториями начинающегося с $N$ частиц однородного ветвящегося процесса Гальтона-Ватсона с пуассоновским распределением числа прямых потомков каждой частицы. Для подмножества траекторий, число вершин которых не превосходит $n$, найдены предельные распределения максимального объема дерева при $N, n \rightarrow \infty$ и различном поведении параметра распределения числа потомков.

Работа поддержана Российским Фондом Фундаментальных Исследований, грант 13-01-00009, и Программой стратегического развития Петрозаводского государственного университета.

Ключевые слова: лес Гальтона-Ватсона, максимальный объем дерева, предельные теоремы.

В $[1,2]$ рассматривалось множество $F_{N, n}$ лесов с помеченными вершинами, содержащих $N$ корневых деревьев и $n$ некорневых вершин. На этом множестве задавалось равномерное распределение вероятностей, и для таких случайных лесов были получены предельные распределения максимального объема дерева при различном характере стремления $N$ и $n$ к бесконечности. Позднее, в [3], аналогичная задача была решена для так называемых лесов Гальтона-Ватсона, т.е. случайных лесов, образованных траекториями докритического или критического процесса Гальтона-Ватсона, имеющего $N$ начальных частиц. Понятно, что такой процесс распадается на $N$ независимых процессов, начинающихся с одной частицы, и множество всех возможных его траекторий бесконечно. В [3] рассматривалось подмножество этого множества, в котором число частиц, существовавших за все время эвол+юции процесса, равно $N+n$. В таком подмножестве число реализаций процесса уже конечно, а распределение вероятностей естественным образом индуцируется ветвящимся процессом.

Пусть процесс Гальтона-Ватсона начинается с $N$ частиц, а число прямых потомков каждой частицы имеет распределение Пуассона с параметром $\lambda, 0<\lambda \leqslant 1$. Заметим, что подмножество траекторий такого процесса, содержащих $N+n$ вершин, совпадает с $F_{N, n}$. Обозначим через $\xi_{1}, \ldots, \xi_{N}$ числа частиц, существовавших

*Место работы: Институт прикладных математических исследований Карельского научного центра PAH, e-mail: pavlov@krc.karelia.ru

** Место работы: Институт прикладных математических исследований Карельского научного центра PAH, e-mail: cher@krc.karelia.ru 
за все время эволюции в подпроцессах, начинающихся с частиц $1, \ldots, N$ соответственно. Легко видеть, что эти случайные величины определяют объемы деревьев леса Гальтона-Ватсона за все время эволюции процесса. Известно [4], что $\xi_{1}, \ldots, \xi_{N}$ имеют распределение Бореля-Таннера:

$$
p_{k}=\mathbf{P}\left\{\xi_{i}=k\right\}=\frac{(\lambda k)^{k-1}}{k !} e^{-\lambda k}, \quad k=1,2, \ldots, \quad 0<\lambda \leqslant 1 .
$$

Далее будем рассматривать подмножество траекторий процесса, удовлетворяющих условию $\xi_{1}+\ldots+\xi_{N} \leqslant n$. Обозначим через $\eta_{1}, \ldots, \eta_{N}$ случайные величины, равные объемам деревьев в лесе из этого подмножества. Очевидно, что справедливо равенство

$$
\begin{gathered}
\mathbf{P}\left\{\eta_{1}=k_{1}, \ldots, \eta_{N}=k_{N}\right\} \\
=\mathbf{P}\left\{\xi_{1}=k_{1}, \ldots, \xi_{N}=k_{N} \mid \xi_{1}+\ldots+\xi_{N} \leqslant n\right\} .
\end{gathered}
$$

Введем случайную величину $\eta_{(N)}$, равную максимальному объему дерева в лесе, т.e.

$$
\eta_{(N)}=\max _{1 \leqslant i \leqslant N} \eta_{i}
$$

В теоремах 1-5 найдены предельные распределения случайной величины $\eta_{(N)}$.

Теорема 1. Пусть $N \rightarrow \infty, N \sqrt{1-\lambda}-n(1-\lambda)^{3 / 2} \leqslant C \sqrt{\lambda N}, 0 \leqslant C<\infty, r \geqslant 5$, $N p_{r-1} \rightarrow \infty, N p_{r} \rightarrow \alpha$, где $\alpha-$ некоторая неотрицательная постоянная. Тогда

$$
\mathbf{P}\left\{\eta_{(N)}=r\right\}=1-e^{-\alpha}+o(1), \quad \mathbf{P}\left\{\eta_{(N)}=r-1\right\}=e^{-\alpha}+o(1) .
$$

Теорема 2. Пусть $N \rightarrow \infty, N \sqrt{1-\lambda}-n(1-\lambda)^{3 / 2} \leqslant C \sqrt{N}, 0 \leqslant C<\infty, N p_{r} \rightarrow \alpha$, где $\alpha$ - некоторое положительное число, $0<\lambda_{1} \leqslant \lambda \leqslant \lambda_{2}<1$. Тогда для любого фиксированного $k$

$$
\mathbf{P}\left\{\eta_{(N)} \leqslant r+k\right\} \rightarrow \exp \left\{-\frac{\alpha\left(\lambda e^{1-\lambda}\right)^{k+1}}{1-\lambda e^{1-\lambda}}\right\}
$$

Теорема 3. Пусть $N \rightarrow \infty, \lambda \rightarrow 1,(1-\lambda) N \rightarrow \infty, N \sqrt{1-\lambda}-n(1-\lambda)^{3 / 2} \leqslant C \sqrt{N}$, $0 \leqslant C<\infty$. Тогда для любого фиксированного $z>0$

$$
\mathbf{P}\left\{\eta_{(N)} \beta-u \leqslant z\right\} \rightarrow e^{-e^{-z}},
$$

где $\beta=-\ln \lambda+\lambda-1$, а и выбрано так, что $N \sqrt{\beta} u^{-3 / 2} e^{-u}=\sqrt{2 \pi}$.

Теорема 4. Пусть $N \rightarrow \infty,(1-\lambda) N \rightarrow \gamma, 0<\gamma<\infty, n / N^{2} \geqslant C>0$. Тогда для любого фиксированного $z>0$

$$
\mathbf{P}\left\{\frac{\eta_{(N)}}{N^{2}} \leqslant z\right\}=e^{-Q(z, \gamma)} \frac{\int_{0}^{n / N^{2}} g_{z}(x) d x}{\int_{0}^{n / N^{2}} g(x) d x}(1+o(1)),
$$

где

$$
Q(z, \gamma)=\sqrt{\frac{2}{\pi z}} e^{-z \gamma^{2} / 2}-\gamma \sqrt{\frac{2}{\pi}} \int_{\gamma \sqrt{z}}^{\infty} e^{-y^{2} / 2} d y
$$




$$
g(x)=\frac{1}{x \sqrt{2 \pi x}} \exp \left\{\gamma-\frac{\gamma^{2} x}{2}-\frac{1}{2 x}\right\},
$$

a $g_{z}(x)$ - плотность распределения с характеристической функцией

$$
\exp \left\{\gamma-\sqrt{\gamma^{2}-2 i t}+Q(z, \gamma)\right\}\left(1-\frac{1}{\sqrt{2 \pi}} \int_{z}^{\infty} y^{-3 / 2} e^{i t y-\gamma^{2} y / 2} d y\right) \text {. }
$$

Теорема 5. Пусть $N \rightarrow \infty,(1-\lambda) N=o(1), n / N^{2} \geqslant C>0$. Тогда справедливъ следующие утверждения.

1. Если $n / N^{2} \rightarrow \alpha, 0<\alpha<\infty$, то для любого фиксированного $z>0$

$$
\mathbf{P}\left\{\frac{\eta_{(N)}}{N^{2}} \leqslant z\right\} \rightarrow 1+\frac{\sum_{k=1}^{[\alpha / z]} \frac{(-1)^{k}}{k !} \int_{k z}^{\alpha} I_{k}(z, y) d y}{\int_{0}^{\alpha} y^{-3 / 2} e^{-1 / 2 y} d y},
$$

гдe

$$
\begin{gathered}
I_{k}(z, y)=\int_{x_{k}(z, y)} \frac{\exp \left\{-1 / 2\left(y-x_{1}-\ldots-x_{k}\right)\right\} d x_{1} \ldots d x_{k}}{(2 \pi)^{k / 2}\left(x_{1} \ldots x_{k}\left(y-x_{1}-\ldots-x_{k}\right)\right)^{3 / 2}} \\
x_{k}(z, y)=\left\{x_{i} \geqslant z, i=1, \ldots, k, x_{1}+\ldots+x_{i} \leqslant y\right\}, \quad k=1,2, \ldots
\end{gathered}
$$

2. Если $n / N^{2} \rightarrow \infty$, то для любого фиксированного $z>0$

$$
\mathbf{P}\left\{\frac{\eta_{(N)}}{N^{2}} \leqslant z\right\} \rightarrow 1+\frac{1}{\sqrt{2 \pi}} \sum_{k=1}^{\infty} \frac{(-1)^{k}}{k !} \int_{k z}^{\infty} I_{k}(z, y) d y
$$

Ниже приводятся леммы 1-10, с помощью которых доказаны теоремы 1-5.

Лемма 1. Справедливо равенство

$$
\mathbf{P}\left\{\eta_{(N)} \leqslant r\right\}=\left(1-P_{r}\right)^{N} \frac{\mathbf{P}\left\{\zeta_{N}^{(r)} \leqslant n\right\}}{\mathbf{P}\left\{\zeta_{N} \leqslant n\right\}},
$$

где

$$
P_{r}=\mathbf{P}\left\{\xi_{1}>r\right\}, \quad \zeta_{N}=\xi_{1}+\ldots+\xi_{N}, \quad \zeta_{N}^{(r)}=\xi_{1}^{(r)}+\ldots+\xi_{N}^{(r)},
$$

а случайные величины $\xi_{1}^{(r)}, \ldots, \xi_{N}^{(r)}$ независимы и имеют распределение

$$
\mathbf{P}\left\{\xi_{i}^{(r)}=k\right\}=\mathbf{P}\left\{\xi_{1}=k \mid \xi_{1} \leqslant r\right\}, \quad k=1,2, \ldots, \quad i=1, \ldots, N .
$$

Доказательство. Соотношение (2) означает, что для случайных величин $\xi_{1}, \ldots, \xi_{N}$ и $\eta_{1}, \ldots, \eta_{N}$ выполнены менее жесткие условия, чем в обобщенной схеме размещения частиц по ячейкам [5], где $\xi_{1}+\ldots+\xi_{N}=n$. В статье [6] показано, что из $(2)$ следует (7).

Лемма 2. Если $N \rightarrow \infty, N p_{r-1} \rightarrow \infty, N p_{r} \rightarrow \alpha$, где $\alpha-$ некоторая неотрицательная постоянная, то

$$
N P_{r} \rightarrow 0, N P_{r-1} \rightarrow \alpha, N P_{r-2} \rightarrow \infty
$$


Доказательство. Учитывая (1), находим, что

$$
p_{r} / p_{r-1}=\lambda e^{-1}(r /(r-1))^{r-2},
$$

а из условий леммы получаем соотношение $p_{r} / p_{r-1} \rightarrow 0$. Отсюда следует, что

$$
\lambda \rightarrow 0 \text {. }
$$

С помощью (1) и формулы Стирлинга

$$
r !=\sqrt{2 \pi} r^{r+1 / 2} e^{-r+\delta(r)}, \quad 0<\delta(r)<1 / 12 r,
$$

нетрудно показать, что

$$
N P_{r-1}=N p_{r}(1+\varepsilon(\lambda))
$$

где

$$
\varepsilon(\lambda) \leqslant 2 \sum_{k=r+1}^{\infty}\left(\lambda e^{1-\lambda}\right)^{k-r} \rightarrow 0
$$

Следовательно,

$$
N P_{r-1} \rightarrow \alpha
$$

Отсюда и из условий леммы получаем, что

$$
N P_{r-2}=N P_{r-1}+N p_{r-1} \rightarrow \infty, \quad N P_{r}=N P_{r-1}-N p_{r} \rightarrow 0 .
$$

Лемма доказана.

Лемма 3. Пусть $N \rightarrow \infty, N p_{r} \rightarrow \alpha$, где $\alpha$ - некоторая положительная постоянная, $0<\lambda_{1} \leqslant \lambda \leqslant \lambda_{2}<1$. Тогда для любого иелого фиксированного $k$

$$
N P_{r+k}=\alpha \frac{\left(\lambda e^{1-\lambda}\right)^{k+1}}{1-\lambda e^{1-\lambda}}(1+o(1))
$$

Доказательство. Из условий леммы следует, что

$$
r \rightarrow \infty
$$

и с помощью (1) и (10) находим, что для любого $l \geqslant 1$

$$
p_{r+l} / p_{r}=(1+l / r)^{-3 / 2}\left(\lambda e^{1-\lambda}\right)^{l}(1+O(1 / r)) .
$$

Используя это соотношение, нетрудно показать, что для любого целого $A>k+1$ выполнено равенство

$$
N P_{r+k}=N p_{r}\left(\sum_{l=k+1}^{A}\left(\lambda e^{1-\lambda}\right)^{l}(1+O(1 / r))+\delta(\lambda)\right),
$$

где

$$
\delta(\lambda) \leqslant 2 \sum_{l=A+1}^{\infty}\left(\lambda e^{1-\lambda}\right)^{l}
$$

Поскольку $\lambda e^{1-\lambda}<1$ при $0<\lambda<1$, величину $\delta(\lambda)$ можно сделать сколь угодно малой, выбрав $A$ достаточно большим. Отсюда и из (12) следует утверждение леммы. 
Лемма 4. Пусть $N \rightarrow \infty, \lambda \rightarrow 1, N(1-\lambda) \rightarrow \infty, r=(u+z) / \beta+O(1)$, где $z-$ фиксированное положительное число, $\beta=-\ln \lambda+\lambda-1$, а и выбрано так, что $N \sqrt{\beta} u^{-3 / 2} e^{-u}=\sqrt{2 \pi}$. Тогда

$$
N P_{r}=e^{-z}(1+o(1))
$$

Доказательство. Легко видеть, что в условиях леммы выполнено (11). Используя (1) и (10), получаем, что

$$
P_{r}=\frac{1}{\lambda \sqrt{2 \pi}} \sum_{k=r+1}^{\infty} k^{-3 / 2} \exp \{k(\ln \lambda-\lambda+1)-\delta(k)\}
$$

где $e^{-\delta(k)}=1+O(1 / r)$ равномерно по $k$. Следовательно,

$$
P_{r}=\frac{1+O(1 / r)}{\lambda \sqrt{2 \pi}} \sum_{k=r+1}^{\infty} k^{-3 / 2} e^{-\beta k}
$$

Несложно проверить, что

$$
\int_{r+1}^{\infty} x^{-3 / 2} e^{-\beta x} d x<\sum_{k=r+1}^{\infty} k^{-3 / 2} e^{-\beta k}<\int_{r}^{\infty} x^{-3 / 2} e^{-\beta x} d x
$$

Поскольку

$$
\int_{r}^{r+1} x^{-3 / 2} e^{-\beta x} d x \leqslant r^{-3 / 2} e^{-\beta r},
$$

из (13) находим, что

$$
P_{r}=\frac{\sqrt{\beta}(1+O(1 / r))}{\lambda \sqrt{2 \pi}} \int_{\beta r}^{\infty} y^{-3 / 2} e^{-y} d y+O\left(r^{-3 / 2} e^{-\beta r}\right) .
$$

Легко видеть, что

$$
\beta=\frac{(1-\lambda)^{2}}{2}(1+o(1)) .
$$

Отсюда и из условий леммы следуют соотношения

$$
u \rightarrow \infty, \quad \beta r \rightarrow \infty .
$$

Тогда

$$
\int_{\beta r}^{\infty} y^{-3 / 2} e^{-y} d y=(\beta r)^{-3 / 2} e^{-\beta r}(1+o(1))
$$

и выполнено равенство

$$
P_{r}=\sqrt{\frac{\beta}{2 \pi}}(u+z)^{-3 / 2} e^{-(u+z)}(1+o(1))=N^{-1} e^{-z}(1+o(1)),
$$

откуда получаем утверждение леммы. 
Лемма 5. Пусть $N \rightarrow \infty, r+1=z N^{2}, 0<z<\infty,(1-\lambda) N \rightarrow \gamma, 0 \leqslant \gamma<\infty$. Тогда

$$
N P_{r}=Q(z, \gamma)(1+o(1))
$$

где $Q(z, \gamma)$ определено в (3).

Доказательство. Используя (1) и (10), находим, что при $\lambda=1$

$$
\mathbf{P}_{r}=\frac{1+o(1)}{\sqrt{2 \pi}} \sum_{k=r+1}^{\infty} k^{-3 / 2}=\frac{1+o(1)}{N \sqrt{2 \pi}} \int_{z}^{\infty} x^{-3 / 2} d x=\frac{1+o(1)}{N \sqrt{\pi z / 2}}
$$

и утверждение леммы доказано.

Пусть $\lambda \neq 1$. С помощью (1) и (10) несложно показать, что имеет место соотношение

$$
P_{r}=\frac{1+o(1)}{\sqrt{2 \pi}} \sum_{k=r+1}^{\infty} k^{-3 / 2} \exp \left\{-\frac{k d^{2}}{2 N^{2}}-\frac{k d^{3}}{3 N^{3}}(1+o(1))\right\}, \quad d=(1-\lambda) N .
$$

Заменяя суммирование интегрированием, получаем, что

$$
\begin{gathered}
P_{r}=\frac{1+o(1)}{N \sqrt{2 \pi}} \int_{z}^{\infty} x^{-3 / 2} \exp \left\{-\frac{x d^{2}}{2}-\frac{x d^{3}}{3 N}(1+o(1))\right\} d x \\
=\frac{1+o(1)}{N \sqrt{2 \pi}}\left(\int_{z}^{A} x^{-3 / 2} \exp \left\{-\frac{x d^{2}}{2}-\frac{x d^{3}}{3 N}(1+o(1))\right\} d x+I_{1}\right),
\end{gathered}
$$

где

$$
I_{1}=\int_{A}^{\infty} x^{-3 / 2} \exp \left\{-\frac{x d^{2}}{2}-\frac{x d^{3}}{3 N}(1+o(1))\right\} d x \leqslant \int_{\varepsilon N}^{\infty} x^{-3 / 2} d x \leqslant \frac{C_{1}}{\sqrt{A}},
$$

и выбором $A$ величину $I_{1}$ можно сделать сколь угодно малой. Символы $C_{1}, C_{2}, \ldots$ здесь и далее означают некоторые положительные постоянные. Нетрудно видеть, что при $z \leqslant x \leqslant A$ выполнено соотношение

$$
\exp \left\{-\frac{x d^{3}}{3 N}(1+o(1))\right\}=1-r(x), \quad \text { где } r(x) \leqslant C_{2} x / N
$$

Поэтому

$$
\begin{gathered}
P_{r}=\frac{1+o(1)}{N \sqrt{2 \pi}}\left(\int_{z}^{A} x^{-3 / 2} e^{-x d^{2} / 2} d x+o(1)\right) \\
=\frac{1+o(1)}{N \sqrt{2 \pi}} \int_{z}^{\infty} x^{-3 / 2} e^{-x d^{2} / 2} d x+o(1 / N) .
\end{gathered}
$$

Отсюда с помощью замены переменной $x=y^{2} / d^{2}$ и интегрирования по частям несложно получить утверждение леммы при $\lambda \neq 1$.

Введем обозначения

$$
m=\mathbf{E} \xi_{1}, \quad \sigma^{2}=\mathbf{D} \xi_{1}
$$


Лемма 6. Пусть $N \rightarrow \infty, \lambda^{3}(1-\lambda) N \rightarrow \infty$. Тогда распределение случайной величины $\left(\zeta_{N}-m N\right) /(\sigma \sqrt{N})$ слабо сходится к стандартному нормальному закону.

Доказательство. Обозначим через $\varphi(t)$ характеристическую функцию случайной величины $\xi_{1}$. Легко проверить, что при $t \rightarrow 0$

$$
\varphi(t)=1+i t m-\left(\sigma^{2}+m^{2}\right) t^{2} / 2+O\left(t^{3}(1-\lambda)^{-5}\right),
$$

где

$$
m=1 /(1-\lambda), \quad \sigma^{2}=\lambda /(1-\lambda)^{3} .
$$

С помощью (16) нетрудно показать, что для характеристической функции $\psi(t)$ случайной величины $\left(\zeta_{N}-m N\right) /(\sigma \sqrt{N})$ при любом фиксированном $t$ выполнено соотношение

$$
\ln \psi(t)=-\frac{t^{2}}{2}+O\left(\frac{1}{\sqrt{N \lambda^{3}(1-\lambda)}}\right)=-\frac{t^{2}}{2}+o(1) .
$$

Отсюда следует утверждение леммы.

Лемма 7. Пусть $N \rightarrow \infty,(1-\lambda) N \rightarrow \gamma$, где $\gamma-$ некоторая неотрицательная постоянная. Тогда распределение суммы $\zeta_{N} / N^{2}$ слабо сходится $\kappa$ распределению вероятностей с плотностъю (4).

Доказательство. Рассмотрим ветвящийся процесс Гальтона - Ватсона, начинающийся с одной частицы, в котором число прямых потомков каждой частицы имеет распределение Пуассона с параметром $\lambda$ :

$$
p_{k}(\lambda)=\frac{\lambda^{k}}{k !} e^{-\lambda}, \quad k=0,1, \ldots
$$

Обозначим через $F(z)$ производящую функцию этого распределения:

$$
F(z)=\sum_{k=0}^{\infty} p_{k}(\lambda) z^{k}
$$

Пусть $\nu$ - случайная величина, равная общему числу частиц, существовавших в процессе до его вырождения. Введем производящую функцию

$$
f(z)=\sum_{l=0}^{\infty} \mathbf{P}\{\nu=l\} z^{l}
$$

Заметим, что $f\left(e^{i t}\right)=\varphi(t)$, где $\varphi(t)$ - характеристическая функция случайной величины $\xi_{1}$. Справедливо равенство [5]:

$$
f(z)=z F(f(z)) .
$$

Отсюда с помощью разложения $F(f(z))$ по формуле Тейлора в окрестности точки $f(z)=1$ находим, что

$$
z\left(\lambda^{2} / 2+\varepsilon(z)\right)(f(z)-1)^{2}+z \lambda(f(z)-1)+z-f(z)=0,
$$

где $\varepsilon(z) \rightarrow 0$ при $f(z) \rightarrow 1$. Учитывая, что для производящей функции выполняется условие $0 \leqslant f(z) \leqslant 1$, из этого квадратного уравнения получаем соотношение

$$
f(z)=1+(1-z+(1-\lambda) z)(1+o(1))-\sqrt{2(1-z)+(1-\lambda)^{2} z+o(1-z)} .
$$


Используя это равенство, для любого фиксированного $t$ нетрудно показать, что при $\lambda=1$

$$
N \ln f\left(e^{i t / N^{2}}\right) \rightarrow-\sqrt{-2 i t},
$$

а при $\lambda \neq 1$

$$
N \ln f\left(e^{i t / N^{2}}\right) \rightarrow \gamma-\sqrt{\gamma^{2}-2 i t} .
$$

Отсюда и из теоремы непрерывности следует утверждение леммы, поскольку $\exp \left\{\gamma-\sqrt{\gamma^{2}-2 i t}\right\}$ является характеристической функцией распределения вероятностей с плотностью (4) [7].

Лемма 8. Пусть $N \rightarrow \infty$ и выполнено одно из следующих условии:

1. $N p_{r-1} \rightarrow \infty, N p_{r} \rightarrow \alpha$, где $\alpha$ - некоторая неотрицательная постоянная, $r \geqslant 5$

2. $N p_{r} \rightarrow \alpha$, где $\alpha$ - некоторая положительная постоянная, $0<\lambda_{1} \leqslant \lambda \leqslant$ $\lambda_{2}<1$

3. $\lambda \rightarrow 1, N(1-\lambda) \rightarrow \infty, r=(u+z) / \beta+O(1)$, где $z-\oint$ - $\rightarrow$ ксированное положительное число, $\beta=-\ln \lambda+\lambda-1$, а и выбрано так, что $N \sqrt{\beta} u^{-3 / 2} e^{-u}=\sqrt{2 \pi}$. Тогда распределение случайной величинь $\left(\zeta_{N}^{(r)}-m N\right) /(\sigma \sqrt{N})$ слабо сходится $\kappa$ стандартному нормальному закону.

Доказательство. Пусть $\psi_{r}(t)$ - характеристическая функция случайной величины $\left(\zeta_{N}^{(r)}-m N\right) /(\sigma \sqrt{N})$. Нетрудно видеть, что

$$
\psi_{r}(t)=\frac{\psi(t)}{\left(1-P_{r}\right)^{N}}\left(1-\varphi^{-1}\left(\frac{t}{\sigma \sqrt{N}}\right) \sum_{k=r+1}^{\infty} p_{k} e^{-i t k /(\sigma \sqrt{N})}\right)^{N},
$$

где $\varphi(t), \quad \psi(t)$ - характеристические функции случайных величин $\xi_{1}$ и $\left(\zeta_{N}-m N\right) /(\sigma \sqrt{N})$ соответственно. Кроме того,

$$
\sum_{k=r+1}^{\infty} p_{k} e^{-i t k /(\sigma \sqrt{N})}=P_{r}+q_{r}(t)
$$

где

$$
\left|q_{r}(t)\right|=\left|\sum_{k=r+1}^{\infty} p_{k}\left(1-e^{-i t k /(\sigma \sqrt{N})}\right)\right| \leqslant \frac{|t|}{\sigma \sqrt{N}} \sum_{k=r+1}^{\infty} k p_{k} .
$$

С помощью (1) и (10) можно показать, что

$$
\sum_{k=r+1}^{\infty} k p_{k} \leqslant C_{3} r p_{r} \sum_{k=r+1}^{\infty}\left(\lambda e^{1-\lambda}\right)^{k-r} .
$$

Пусть выполнено условие 1 леммы. Тогда справедливо (9) и, учитывая (17), (21) и соотношение $N p_{r} \rightarrow \alpha$, из (20) находим, что

$$
\left|q_{r}(t)\right| \leqslant C_{4} \frac{r \sqrt{\lambda}}{N^{3 / 2}} .
$$

Очевидно, что при фиксированном $r$ имеет место оценка

$$
q_{r}(t)=o(1 / N) .
$$

4 Дискретная математика, т. 26 № 3 
При $r \rightarrow \infty$ с помощью (1), (10) и соотношения $N p_{r-1} \rightarrow \infty$ получаем, что $\ln N / r \rightarrow \infty$, и из (22) следует (23). Несложно проверить, что выполнено условие $\lambda^{3} N \rightarrow \infty$, и утверждение леммы следует из (18), (19), (23) и лемм 2,6 .

При выполнении условия 2 леммы, используя (10), (11) и условие $N p_{r} \rightarrow \alpha, \alpha>0$, нетрудно показать, что $0<C_{5} \leqslant \ln N / r \leqslant C_{6}<\infty$, и из (20), (21) следует (23). Из $(18),(19),(23)$ и лемм 3,6 получаем утверждение леммы.

Пусть выполнено условие 3. Аналогично (13) можно показать, что

$$
\sum_{k=r+1}^{\infty} k p_{k}=\frac{1+O(1 / r)}{\lambda \sqrt{2 \pi}} \sum_{k=r+1}^{\infty} \frac{e^{-\beta k}}{\sqrt{k}} .
$$

Отсюда находим, что

$$
\sum_{k=r+1}^{\infty} k p_{k}=\frac{1+O(1 / r)}{\lambda \sqrt{2 \pi \beta}} \int_{\beta r}^{\infty} \frac{e^{-x}}{\sqrt{x}} d x+O\left(\frac{e^{-\beta r}}{\sqrt{r}}\right)=\frac{e^{-\beta r}}{\beta \sqrt{2 \pi r}}(1+o(1)) .
$$

Из (15) и равенства $N \sqrt{\beta} u^{-3 / 2} e^{-u}=\sqrt{2 \pi}$ следует, что $u=\ln (N \sqrt{\beta})(1+o(1))$, и с помощью $(14),(17),(24)$ и соотношения $\beta r=u+z+o(\beta))$ из (20) получаем, что для любого фиксированного $t$

$$
\begin{gathered}
\left|q_{r}(t)\right| \leqslant \frac{C_{7} e^{-u}(1-\lambda)^{3 / 2}}{\sqrt{\beta u N}} \\
=\frac{C_{7} \sqrt{2 \pi} u(1-\lambda)^{3 / 2}}{\beta N \sqrt{N}} \leqslant \frac{C_{8} \ln (N \sqrt{\beta})}{N(N \sqrt{\beta})^{1 / 2}},
\end{gathered}
$$

т.е. справедливо (23). Отсюда, из (18), (19) и лемм 4, 6 следует утверждение леммы. Лемма 9. Пусть $N \rightarrow \infty, r+1=z N^{2},(1-\lambda) N \rightarrow \gamma$, где $z, \gamma-$ некоторье положительные постояные. Тогда распределение $\zeta_{N}^{(r)} / N^{2}$ слабо сходится $к$ распределению вероятностей с характеристической функцией (5).

Доказательство. Нетрудно видеть, что характеристическая функция $\psi_{N}^{(r)}(t)$ суммы $\zeta_{N}^{(r)} / N^{2}$ имеет вид

$$
\psi_{N}^{(r)}(t)=\frac{1}{\left(1-P_{r}\right)^{N}}\left(\varphi\left(\frac{t}{N^{2}}\right)-\sum_{k=r+1}^{\infty} p_{k} e^{-i t k / N^{2}}\right)^{N},
$$

где $\varphi(t)$ - характеристическая функция случайной величины $\xi_{1}$. Аналогично доказательству леммы 5 можно проверить, что при любом фиксированном $t$

$$
\sum_{k=r+1}^{\infty} p_{k} e^{i t k / N^{2}}=\frac{1+o(1)}{N \sqrt{2 \pi}} \int_{z}^{\infty} y^{-3 / 2} e^{i t y} e^{-\frac{y}{2}((1-\lambda) N)^{2}} d y+o\left(\frac{1}{N}\right) .
$$

В доказательстве леммы 7 показано, что

$$
\varphi^{N}\left(t / N^{2}\right)=\exp \left\{\gamma-\sqrt{\gamma^{2}-2 i t}\right\}(1+o(1) .
$$

Используя (25)-(27) и лемму 5, получаем соотношение

$$
\psi_{N}^{(r)}(t)=\frac{e^{\gamma-\sqrt{\gamma^{2}-2 i t}}}{e^{-Q(z, \gamma)}}\left(1-\frac{1}{\sqrt{2 \pi}} \int_{z}^{\infty} y^{-3 / 2} e^{-\gamma^{2} y / 2} e^{i t y} d y\right)(1+o(1)) .
$$

Отсюда и из теоремы непрерывности следует утверждение леммы. 
Лемма 10. Пусть $N \rightarrow \infty, r+1=z N^{2}$, где $z$ - некоторая положительная постоянная, $(1-\lambda) N=o(1)$. Тогда распределение случайной величины $\zeta_{N}^{(r)} / N^{2}$ слабо сходится $к$ распределению вероятностей с плотностъю

$$
g(y)=\frac{e^{\sqrt{2 / \pi z}}}{\sqrt{2 \pi}} \sum_{k=0}^{\infty} \frac{(-1)^{k}}{k !} I_{k}(z, y)
$$

где $I_{0}(z, y)=y^{-3 / 2} e^{-1 / 2 y}$, а $I_{k}(z, y), k=1,2, \ldots$, определенъ в $(6)$.

Доказательство. Из (26) следует, что при $\lambda=1$

$$
\sum_{k=r+1}^{\infty} p_{k} e^{i t k / N^{2}}=\frac{1+o(1)}{N \sqrt{2 \pi}} \int_{z}^{\infty} y^{-3 / 2} e^{i t y} d y=\frac{E(t, z)}{N}(1+o(1)),
$$

где

$$
E(t, z)=\frac{1}{\sqrt{2 \pi}} \int_{z}^{\infty} y^{-3 / 2} e^{i t y} d y
$$

Пусть $\lambda \neq 1, d=(1-\lambda) N$. Разобьем область интегрирования в (26) на две части: $\{z \leqslant y \leqslant A\},\{A \leqslant y \leqslant \infty\}$, где величина $A$ может быть выбрана сколь угодно большой, и рассмотрим каждый интеграл отдельно.

Очевидно, что

$$
\left|\int_{A}^{\infty} y^{-3 / 2} e^{-d^{2} y / 2} e^{i t y} d y\right| \leqslant \int_{A}^{\infty} y^{-3 / 2} d y=\frac{2}{\sqrt{A}} .
$$

При $z \leqslant y \leqslant A$ выполнено равенство $e^{-d^{2} y / 2}=1-d^{2} y / 2(1+o(1))$. Следовательно,

$$
\begin{gathered}
\int_{z}^{A} y^{-3 / 2} e^{-d^{2} y / 2} e^{i t y} d y=\int_{z}^{A} y^{-3 / 2} e^{i t y} d y-\frac{d^{2}}{2}(1+o(1)) \int_{z}^{A} y^{-1 / 2} e^{i t y} d y \\
=\int_{z}^{\infty} y^{-3 / 2} e^{i t y} d y-\int_{A}^{\infty} y^{-3 / 2} e^{i t y} d y-\frac{d^{2}}{2}(1+o(1)) \int_{z} y^{-1 / 2} e^{i t y} d y,
\end{gathered}
$$

где

$$
\left|\int_{A}^{\infty} y^{-3 / 2} e^{i t y} d y\right| \leqslant \frac{2}{\sqrt{A}}, \quad\left|\int_{z}^{A} y^{-1 / 2} e^{i t y} d y\right| \leqslant 2 \sqrt{A} .
$$

Таким образом, из $(26)$ и $(29)$ находим, что если $d \rightarrow 0$, то выполнено соотношение (28).

Легко видеть, что

$$
P_{r}=\sum_{k=r+1}^{\infty} p_{k}=\frac{E(0, z)}{N}(1+o(1)) .
$$

Из леммы 7 следует (27), и, используя (25), (27), (28), для характеристической функции $\psi_{N}^{(r)}(t)$ суммы $\zeta_{N}^{(r)} / N^{2}$ получаем равенство

$$
\psi_{N}^{(r)}(t)=\exp \{\sqrt{-2 i t}+E(0, z)-E(t, z)\}(1+o(1)) .
$$


Учитывая, что $E(0, z)=\sqrt{2 / \pi z}$, аналогично доказательству леммы 3.3 .1 [5] можно показать, что справедливо утверждение леммы.

Докажем теперь теоремы 1-5.

В условиях теоремы 1 справедливо соотношение (9) и, используя (1), легко обнаружить, что $\lambda^{3} N \rightarrow \infty$. Поэтому можно применить лемму 6 , а также равенства (17) и леммы $1,2,8$.

Теорема 2 следует из лемм $1,3,6,8$.

Нетрудно проверить, что при выполнении условий теоремы 3 в силу (17) и лемм 6,8 отношение $\mathbf{P}\left\{\zeta_{N}^{(r)} \leqslant n\right\} / \mathbf{P}\left\{\zeta_{N} \leqslant n\right\}$ стремится к единице. Утверждение теоремы 3 вытекает из лемм 1 и 4 , если величина $r$ в (7) задана условиями леммы 4.

Аналогичным образом легко убедиться, что теорема 4 следует из лемм $1,5,7$ и 9 .

Из леммы 7 находим, что при выполнении условий теоремы 5

$$
\mathbf{P}\left\{\zeta_{N} \leqslant n\right\}=\frac{1}{\sqrt{2 \pi}} \int_{0}^{n / N^{2}} x^{-3 / 2} e^{-1 / 2 x} d x .
$$

Отсюда, из лемм 1, 5, 10 получаем утверждение теоремы 5.

\section{Список литературы}

1. Павлов Ю. Л., "Асимптотическое распределение максимального объема дерева в случайном лесе", Теория вероятн. и ее применен, 22:3 (1977), 523-533.

2. Павлов Ю. Л., "Один случай предельного распределения максимального объема дерева в случайном лесе", Матем. заметки, 5 (1979), 751-760.

3. Pavlov Yu. L., Random forests, VSP, Utrecht, 2000.

4. Севастьянов Б. А., Ветвящиеся процессы, Наука, Москва, 1971.

5. Колчин В. Ф., Случайные отображения, Наука, Москва, 1984.

6. Чупрунов А. Н., Фазекаш И., “Аналог обобщенной схемы размещения. Предельные теоремы для максимального объема ячейки”, Дискрет. матем., 24:3 (2012), 122-129.

7. Оберхеттингер Ф., Преобразования Фуръе распределений и их обращения, Наука, Москва, 1979. 